\title{
INTRODUCCIÓN AL ESTUDIO DE LOS SONETOS DE RUBÉN DARÍO
}

"Ėn poesía, como en toda manifestación artística, no hay nueva escuela, nuevo estilo, si la innovación no alcanza también de nlinera fundamental a la forma...» 1 .

El Modernismo va a traer consigo, como una de sus características esenciales, la libertad en el uso de los metros estróficos, al igual que en el ritmo versal. Por una parte, los poetas modernistas renovarán las formas en desuso, partiendo de Berceo y recorriendo toda la lírica de habla sastellana; por otra, y por medio de una labor consciente y deliberada, traerán nuevas formas: unas creadas por ellos, y ensayadas y conseguiclas otras en la lírica francesa. El Modernismo completa la labor iniciada en el Romanticismo, generaliza formas ya iniciadas tímidamente, inprime carácter de novedad a algunas "viejas» y ensaya mil y mil formas nuevas. Según Leopoldo Díaz, es necesario remontarse a la métrica clásica, y recomienda, en otra ocasión, "hacer versos magníficos $y$ raros".

La labor de los poetas modernistas alcanza a todos los aspectos de la creación poética: al verso y a la estrofa, a los temas y a las series rítmicas... Nuestro estudio se referirá exclusivamente al soneto; a partir (lel verso, llegaremos a analizar la estructura de esta composición, considerando, antes, los temas y la rima de los pies versales. El soneto, que el Romanticismo no habla cultivado, ha sido, sin embargo, la forma preferida por los poetas del siglo xx; pero si el hálito lírico de Bécquer se considera el punto de partida de la poesía contemporánea, sin duda alguna la revolución métrica del Modernismo es la base también de los esquemas rítmicos de la poesía de este siglo.

1 E. DfEz ECHARRI, Métrica modernista: innovaciones y renovaciones. Rewista de Literalura, 1957, XI, p. ioz-120. 
Si consideráramos recogida definitivamente toda la labor poética de Rubén Darío en las últimas Poesias Complctas publicadas ${ }^{1}$, serían I 28 los sonetos escritos por nuestro poeta; hasta I886, Darío escribió 22 sonctos, ensayo de los que incluirá posteriormente en los libros por él organizados, y que pueden considerarse como verdaderas obras maestras. de esta composición ${ }^{2}$.

Los versos prefericlos por Rubén son el octosílabo, el endecasílabo, el alejandrino $y$, en menor proporción, el decasílabo; pero, mientras el primero y el último no sufren grandes transformaciones, el endecasílabo y el alejandrino adquieren una importancia extraordinaria a causa de haber empleado en sus poemas nuestro poeta todas las variantes acentuales de estos versos.

Por lo que se refiere a los sonetos, la mayor parte de los de nuestro. autor están escritos en versos endecasílabos y alcjandrinos, pero encontramos también sonetos en hexasílabos (Mia, p. 569), en heptasílabos (Para Alice de Bolaños, p. 1034; A Francisca p. ro8o), en octosílabos (Para una cubana, p. 563; Para la misma, pp. 563-564; Un soncto para. bebé, pp. 874-875; Toisón, pp. I043-I044), en eneasílabos (El soneto de

1 Poesías completas. Edición de Arfonso Míndez Prancartr y prólogo y apéndice de Antonio Ol,Ivrer Brimás. Madrid, Aguilar, I967.

2 Sobre el soncto pucde encontrarse amplia bibliograffa, hasta la fecha de edición en la obra de Warter Möncir, Das sonett, Heiderberg, 1955, y ell la de Ai,fredo Carbali,o Picazo, Métrica española. Madrid, i956. Consúltese también TOmis Navarro Tomis, Metrica española. Nueva York, 1956; del mismo, Ritmo y armonía en los versos de Dario, en La Torre, x967, núms. 55-56, 1p. 49-69: RAFAII, DF, BALBín, Sistema de ritmica castellana, Madrid, ig68; MARIo FuBINI, Metrica e poesia, Milano, I963 y RUDOI, BAERH, Spanische Verslehre, Tübingen, 1962. Sobre los sonetos de Dario en particular véase J. Sanvenra Molina, Los hexánetros castellanos y en particular los de Rubén Dario, en Anales de la Universidad de Chile, 1935, XCIII, núm. I 8 p. 5-90; E. K. MAPTS, Los primeros sonelos alejandrinos de $R$. D., en Revista Hispanica Moderna, I, 1935, pp. 241-259; del mismo, Innotación e influencia francesit en la métrica de $R$. $D$., en Revista Hispánica Moderna, VI, 1940, pp. I-16; J. F. Síncirez, De la métrica de R. D., en Anales de la Universidad de santo Domingo, 1954, XIX, núms, 69-70, pp. 65-94; S. AGUndo ANDREUT, Este gran don Ramón de las barbas de chivo: Análisis de un soneto de R. D.: hombre y poeta. en Revisfa Iberoamericana, XXV, 1960, núm. I, pp. 7-I 2; I". MaLdonado DE GUEVARA, Un soneto de R. D. (Sobre "Urna votiva"). en Seminario Archivo de Rubén Dario, r96r, núm. 1, pp. 7-12; A. OLrver BeLMAs, En la iiltima pigina de "El Romancero del Cid". Un extraño soneto juvenil de R. D., en Seminario Archivo de Rubén Dario, 1964, núm. 9, pp. 7-9. Una aunplia bibliografia sobre nuestro poeta ha aparecido recientemente, publicada por FEDERICO nE ONfs, en La Torre, núms. 35-36, 1967, pp. 46r-495. 
trece versos, p. 666; ¡Oh Dios!..., p. I092), en decasilabos (Monteviden, p. I067), en dodecasílabos (Simón el bobito, p. 872), en pentadecasílabos (A Francia, pp. 709-7Io), e incluso enplea versos de diferente número de sílabas en una misma composición, como veremos más alelante.

Podemos encontrar todas las variantes acentuales del endecasílabo en los sonetos de Darío, pues su renovación, en cuanto a este versn, es mucho más que la resurrección del olvidado endecasílabo de "gaita gallega»; y en cuanto al alejandrino, "el verso típico del modernismon, según Oliver Bclmás ${ }^{1}$, Rubén lo hace flexible, consigue que pierda su rigidez métrica a base de un desplazaniento de la cesura y de los acentos secundarios. Ya no estará la cesura - que no es un corte sino una ondulación, como dice Maldonado de Gueva ra ${ }^{2}$ - desptés de la séptima sílaba; Rubén la pondrá después de la cuarta, de la novena, ctc., introducirá clos cesuras en el mismo hemistiquio o bien hará triple el alejandrino... ${ }^{3}$.

\section{Temas de los sonetos}

Analizando temáticamente los sonctos, se observa fácilmente que nuestro autor prefería emplearlos sobre todo para lomenajear o cantar a personas, naciones, ciudades, ideas abstractas, etc. Casi el 70 por roo está dedicado a exaltar las virtudes o la obra de un escritor o amigo, 11 país, una ciudad...

Los primeros sonetos tendrán cono fin cantar $A$ los liberales, los sonetos en endecasílabos y siguiendo la forma tradicional del esrjucna rítnico, aunque encontramos, cn uno, una característica especial cn la rima de los versos primero, cuarto, quinto y octavo, que estudiamos más adelante. Con sonetos cantará Rubén a Garcilaso (p. 260), a I cconte de Lisle, Catulle Mondès, Whitman, Palma, Parodi y Mirón en Azul (p1). 537-540); a Berceo, en Prosas profanas (p. 62x); a Góngora y Vclázculez. (pp. 66o-66r), a Cervantes (p. 669) en Cantos deVida y Esperania; a Valle Inclán (p. 763) en El canto crrante y a Juan Ramón y Amado Nerro (pp. In03-I004) en la obra dispersa... Y por otra parte, A Jérez (p. 58); A Bernal (p. 95) y A Juan Diego Braun (p. I7I); a Caupolicán (p. 535); A una cubana (p. 563) a La gitanilla (p. 620); a Bradomín (p. 680); a Charles de Soussens (p. $76 \mathrm{I}$ ); a Un bebé (p. 874; a Ladislao Errázuriz.... (p. 878) y a Máximo Soto Hall (p. 937); a Lohengrin (p. 963); a Parsifal

1 Ese otro Rubén Dario, Barcelona, Aedos, r96o.

2 La función del alejandrino francés en el alejandrino español de R. D., Rezista de Literatura, 1953, IV, pp. 9-58.

3 Véase El endecastlabo castellano y Sobre la historia del alejandrino, de PrDRo HrNRfQUTZ URT̃NA, en Estudios de versificación española, Buchos Aires, iofr. 
(p. 963); a Carlos Ramagosa (p. 974); a Manucl Maldonado (p. I022); a Carrasquilla (p. I033); a Alice (p. I034) y Vargas Vila (p. I037); a D'Halmar (p. 1070) y a Ricardo Pérez (p. ro77)... Por otra parte, a La espiga (p. 6I4); a Los poetas risueños (p. 6r8); a Gaita galaica (p.790); al Cuello blanco (p. 919); al David de Miguel Angel (p. I004); por otra, a Francia (p. 709); a Colombia (p. 938); a España (p. I000), a Bolivia (p. I00o); a Roma (p. 976); por otra, a El ferro-carril (p. I88), a La razón (p. 27), A los piratas (p. 767), etc...

Se puede, efcctivamente, objetar que también Darío escribió ell otras formas estróficas cantos a poetas, amigos, naciones, etc., pero lo que no cabe duda es esta preferencia del soneto, que considero ejemplificada absolutamente, con esta finalidad. Es más; Darío se aparta del soneto para cantar a aquellos a quienes más admiraba y, así, Verlaine y Víctor Hugn, Argentina y Chile, etc., están homenajeados por Darío en largas composiciones: cl cuadro o la apología que hace de esos poetas o esas naciones es demasiado extenso para encerrarlo en catorce versos.

Darío, en la mayoría de los casos, no hace en sus sonetos el retrato del personaje o la obra creada por el personaje a quien canta, así como tampoco describe las bellezas de las naciones o de las ciudades; canta virtudes objetivamente, sin personificarlas en lo cantado, para, en los últimos versos, dirigirse ya a la persona o cosa cantada, personificándola. Véase el soneto dedicado a Carlos Ramagosa:

Entre la barbarie que las garras saca cuando ve el reflejo de sedas fulgentes; entre el que desdora, desluce $y$ ataca, eriza las cerdas y muestra los dientes cuando ve mi gema, mi biombo, mi laca, o mis raras musas, terror de las gentes, terror de la gente calmuca y canaca que mancha la gloria de los continentes..., suele haber un cisne, o un águila bella, o un lirio, o un trueno, o una blanca estrella o un iris que muda la noche que mata, - una espada de oro, o un casco de plata, o una rosa pura, o una noble rosa..., itoda como tu alma, Carlos Ramagosa! (1896).

Normalmente, Darío emplea la tercera persona, ya que las composiciones van dirigidas al lector, pero a veces es a la propia persona cantada a quien se dirige la lectura y entonces emplea la segunda: 


\section{Salvador Díaz Mirón}

Tu cuarteto es cuadriga de águila bravas que aman las tempestades, los océanos; las pesadas tizonas, las férreas clavas. son las armas forjadas para tus manos:

Tu idea tiene cráteres $y$ vierte lavas; del Arte recorriendo montes y llanos, van tus rudas estrofas jamás esclavas, como un tropel de buifalos americanos.

Lo que suena en tu lira lejos resuena, como cuando habla el bóreas, o cuando truena. iHijo del Nuevo Mundo!, la Humanidad oiga, sobre la frente de las naciones, la himnica pompa lírica de tus canciones que saludan triunfantes la libertad. (De Azul.)

Sin pretender hacer una clasificación temática de los sonetos de Dario, diriamos que hay una serie de temas que se repiten con frecuencia y asi, aparte de los sonetos mencionados más arriba, dirigidos a poetas, amigos, naciones, etc., el grupo más importante por su número, habría otros descriptivos (De invierno, p. 536), reflexivos (Creer, p. 95), de recucrdos infantiles o juveniles (Triptico de Nicaragua, pp. Io6I-62), filosóficos (¡Oh, Dios! p. I092)... Rubén escribió sólo cuatro sonetos amorosos: los dos primeros, Magna veritas y $A$ mi Filis (pp. I38-39), cantan un amor perdido, pero, en lugar de hacerlo triste y nostálgicamente o pidiendo que la mujer vuelva a su lado, finalizan un tanto humoristicamente; en el primero dice la mujer al poeta:

Señor poeta, vaya utsté a paseo

jotros hay que me ofrecen mucha plata!

y en el segundo el poeta dice a la mujer:

$Y$ no hay que arrepentirse; a lo hecho, pecho.

Mira, no es broma, con franqueza te hablo;

sigue en tu nuevo anor, y'... ibuen provecho!

Pero al lado de estos, escribió Darío otros dos plenamente amorosos y de un intenso lirismo: Mia (p. 569), y A Francisca (I) (p. I080), a su I'rancisca, a quien Rubén recibe así:

Francisca, tii has venido

en la hora segura;

la mañana es obscura

$y$ está caliente el villo. 


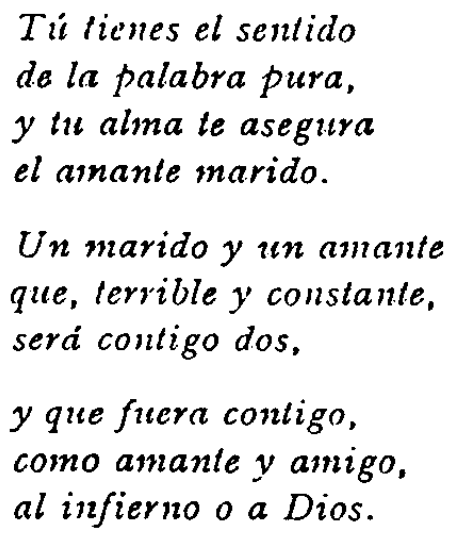

Destacan la enorme belleza $y$ la calidad de tres sonetos, compuestos en los últimos años y que recuerdan mucho la poesía de Machado y Unamuno. Es el primero , Oh Dios! lleno de duda, escéptico y angustiado; el segundo, Pasa y olvida, más escéptico aún que el anterior, y el tercero, lara ir al azul... de una ternura y una emoción lírica extraordinarias. Puede que formalmente no sean de los mejores del poeta nicaragüense; los considero como tuna cima de autenticidad en la línea vital de la poesía de Rubén.

\section{La rima 1}

Los 22 sonetos anteriores a I 886 tienen todos, excepto uno, rima paroxítona o grave; solamente El ferro-carril (p. I88) tiene $\mathrm{I}_{3}$ y I4 con rima proparoxítona o esdrújula ${ }^{2}$.

De I 886 a I 889 , es decir, hasta la aparición de la segunda edición de $1: u^{3}{ }^{3}$, los esquemas rítmicos serán: Simón el bobito, rima oxítona en 2 , 4,6 y 8 al I4; Un soneto para bebé, rima oxítona en $\mathrm{I}, 4,5,8$, I0, I2 y I4; Lastarria, rima oxítona en 4, 8, II y $\mathrm{I}_{4}$; Sonetos americanos, los dos con rima oxítona en $2,4,6,8$, II y I 4 ; La Revolución francesa, los tres primeros sonetos con rima oxítona en los pares, el cuarto en $2,4,6,8$, II y I4; este soneto también con rima proparoxítona en I2 y I3; El ánfora, rima oxítona en los pares.

1 Para todos los aspectos relacionados con la tima debe consultarse Sistema de ritmica castellana, de RAFAEI, DE BALBÍN, pp. 219-50.

2 Emplearemos en nuestro trabajo los términos de rima oxitona, paroxitona y proparoxitona por estimar que son más concretos que los de aguda, llana o esdrujula, y por ser también los más empleados hoy. Consúltese ANronio QUILIs, Sobre la percepción de los versos oxitonos y proparoxitonos en español, RFE, 1967, I, pp. 273-286.

3 Ira primera edición de Azul (1888) no incluía ningún soneto; la segunda aparecio en 1890 con los Snnelos áureos. 
En $A z u l$ los ocho sonetos de la segunda edición del libro alternan la rima paroxítona con la rima oxitona, que aparece con nuchísina frecuencia, y la rima proparoxítona, en el soneto $V e n u s$ con tres versos rimando en -álida (9, I I y I4); es éste el poema de mayor riqueza rítmica del libro y uno de los más ricos musicalmente de toda la producción de Darío: I, 3,5 y 7 tienen rima oxitona; los versos pares, rima paraxitona y 9, II y I3, rima proparoxítona. En cuanto a los restantes sonetos: Caupolicán: rima oxítona en $2,4,6,8$, Ir y I4; De invierno: rima oxítona en los pares; Leconte de Lisle: rima oxítona en 2, 4, 6, 8, I I y I4; Catulle Mendès: rima oxitona en 2, 4, 6, 8, I I y I3; Whitman: rima oxítona en Io, I2 y I4; Ricardo Palma: rima oxftona en pares; Diaz Mirón: rima oxítona en II y I4.

En Prosas profanas decrece la variedad acentual observada en $\mathrm{Azul}$ : aparece la rima proparoxitona en cuatro versos del soneto Para una cubana ( $I, 3,5$ y 7); rima oxítona en los siguientes sonetos: Para la misma: II, I2, I3 y I4; Margarita: 2, 4, 6, 8, Io y I4; Ite misa est: 2, 4, 6, 8, II I 4; El cisne: $2,4,6,8$, II y $x 4$. En los catorce sonetos restantes la rima será paroxítona en todos ellos.

Menos cantidad encontramos aún en Cantos de vida y esperanza; de los dieciséis sonetos del libro únicamente en tres alterma la rima oxítona con la rima paroxítona: Soneto de trece versos: $2,3,6,7,9$, I I y I3; Caracol: II y I4; Urna votiva: II y I4.

Aumentan, sin embargo, en el Canto errante los sonetos con las tres clases de rima: A Francia: rima oxitona en los pares; Esquela a Charles de Soussens: rima oxítona en $2,4,6,8$, ix y I4; Helda: rima oxitona en I, 4, 5, 8, II, I2, I3 y I4. (Este soneto está escrito en francés). Lirica: rima oxitona en II y I4 y proparoxitona en I2 y I3; Los piratas: rima oxítona en II y I4. Los dos únicos sonetos de Poema de Otoño llevan rima oxítona: Ir y I4 de $E_{n}$ casa del doctor... y todos los de Gaita galaica.

Veamos, a continuación, los sonetos de la obra dispersa posteriores a I890 y reunidos todos bajo el título de Del chorro de la fuentc. I,os agrupamos por fechas de composición:

De I890 a I897. En los dos agrupados bajo el título general de IVagneriana y subtitulados Lohengrin y Parsifal tienen rima oxítona en I y I4; en Flor argentina 2, 4, 6, 8, II y I4, y $A$ Suzette en I, 4, 5, 8, 9, ro, I2 y I3. (Este soneto, como Helda, está escrito en francés.)

De 1898 a 1907 . Darío compone dos sonetos no recogidos en sus libros, con rima rica: Amado Nervo, oxitona en los pares, y Flora, con proparoxítona en $\mathrm{I} 2 \mathrm{y} \mathrm{I} 4$.

De igos a Igro. A Carrasquilla...: rima rica oxítona aguila c1l 2. \&, 
6, 8, II y I4; En las constclaciones: rima oxítona en II y I4; $A$ Vargas Vila...: rima oxítona en $2,3,6$ y 7 .

De IgII a I9I4. Desde la variedad acentual de $A z u l$ hasta estos últimos años de la vida del poeta, ha ido decreciendo el número de ejemplos de riqueza rítmica. El Modernismo comenzó métricamente con Azul y allí se dio el gran paso; el mismo Darío con su obra nos lo demuestra. En la última época escribe algunos sonetos; encontramos ejemplos de rima rica también, pero en mucha menor cantidad. $A$ D'Halmar: tiene rima oxítona en II y I4; Puesto que tii me dices: rima oxítona en Io, I2 y I4; A Francisca: en II y I4; iOh Dios!: rima oxítona en las dos rimas de los tercetos. Para ir al Azul: en 2, 3, 6 y 7 .

El último de los sonetos de Rubén Darío, escrito en la Navidad de I9I4, Soneto Pascual, lleva también rima oxítona en ir y I4.

\section{Casos especiales}

E.l primero de los sonetos de Rubén, $A$ los libcrales, presenta una particularidad: riman I con 4 y 5 con $\delta$ en consonante, pero sólo si fonéticamente recurrimos al "seseo":

Porque canttis la eterna Marsell sa
que maldice el poder de los tiranos;
porque alzais ardorosos con las manos
el pendón de la luz con entereza;
porque deseais que caiga la cabeza
de la hidra aristocrálica, y ufanos
dais al pueblo principios soberanos
que destruyen del mal la niebla espesa.

I.o mismo sucede en el soneto que comienza Tras los cucrnos de Jiifilcr.., incluido en el "Apéndice poético" de la última edición de las Poesías Completas (p. I I39):

Montañas que quedasteis lejanas, lagos puros, hovizontes de fuego y rincones obscuros, cafetales floridos, gentes anables, voz

gentil que recordaste mi infancia y de mi infancia luz, gesto, vida, volunlad y fragancia y todas esas cosas que yo no olvido, iadiós! 
En Ante la cstatua de Morazán y en I3 se encuentra, como palabra final del pie versal, "entonces»: para la rima consonante perfecta, Rubén escribió "entonce», $\sin -s \mathrm{y}$, así rima con la última palabra del verso siguiente, «bronce».

Atención especial merece la composición Menéndez. No creo que sea un soneto, aunque se repita como tal en las ediciones de Darío; la única razón de esta catalogación es tener catorce versos, pero soll versos le diez sílabas, y lo más esencial, con el esquema rítmico del romance; la rima es asonante y sólo riman los pares.

Es necesario destacar también un hecho singular: la gran cantidad te ejemplos de rima oxítona o aguda ell el último verso que hemos podido ofrecer: gracias a este acento fuerte, el poena se cierra con una intensidad imposible en la acentuación grave.

Como resumen de este capítulo, puede afirmarse la diversidad acentual de los pies versales en los sonetos de Darío, pero sin que esta diversidad en el uso de la rima rica sea tan intensa cono en las restantes composiciones en otros metros. Hay abundancia de ejemplos de rima oxítona o aguda; muy pocos de rima proparoxítona o esdrújula. Son dignos de mención especial Venus y el cuarto soneto de los dedicados a la Revolución francesa, los dos únicos en que se reúnen las tres clases de rima. He aquí el primero, como ejemplo de la riqueza musical de los sonetos modernistas, y, claro está, de los de Rubén:

En la tranquila noche, mis nostalgias amargas sufria, En busca de quietud, bajé al fresco y callado jardin: En el oscuro cielo, Venus bella temblando lucia, como incrustado en ébano un dorado y divino jazmin.

A mi alma enamorada, una reina oriental parecia que esperaba a su amante, bajo el techo de sucamarin, o que, llevada en hombros, la profunda extensión recorria, triunfante y luminosa, recostada sobre un palanquin.

"Oh reina rubia! - dijele-, mi alma quieve dejar su crisálida $y$ volar hacia ti, y hus labios de fuego besar: y flotar en el nimbo que derrama en tu frente pálida. $y$ en siderales éxtasis no dejarte un momento de amarn. El aire de la noche refrescaba la atmósfera cálida. Venus, desde el abismo, me miraba con triste mirar. (De Azul.)

4. La estructura de los sonetos de Rubén Dario

Atendiendo a la rima de las cláusulas estróficas del soneto, pueden estudiarse la estructura de los de Darín, sus esquemas preforidos. Fin lis 
páginas siguientes, y a base de cuadros, se consicleran todos ellos y los esquenas empleados. "Rima abrazada" designa el esquema tradicional rítmico del soneto en las dos primeras cláustulas estróficas: $\mathrm{ABBA} A \mathrm{ABBA}$, y "rima cruzada" el esquema ABAB:ABAB.

Hasta 1886

A los liberales $I \ldots \ldots \ldots \ldots \ldots \ldots \ldots \ldots$

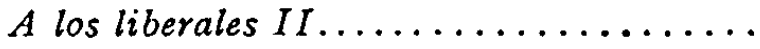

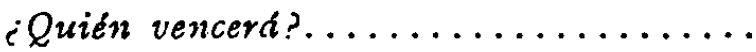

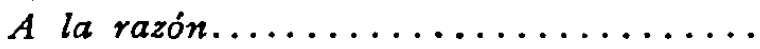

A Jérez.

Ante la estatua de Morazán............

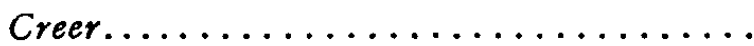

Bernal.

A la Señorita Josefa Dubon..........

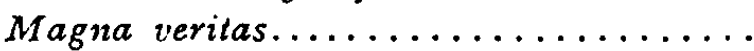

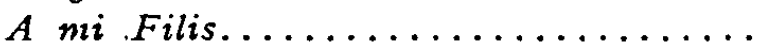

Miel.

El Cantar do los Cantares..............

Triptico $I \ldots \ldots \ldots \ldots \ldots \ldots \ldots \ldots$

Triptico $I I \ldots \ldots \ldots \ldots \ldots \ldots \ldots \ldots$

Triptico $I I I \ldots \ldots \ldots \ldots \ldots \ldots \ldots$

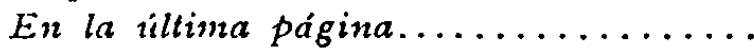

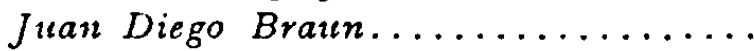

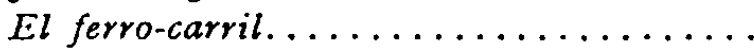

*Dulce como la mieln.................

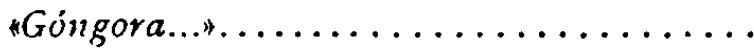

"Quién aparece...?"................

Abrojos (1887)

"¿ Por qué ese orgullo...?"............

De $\quad$ I 886 a 1889

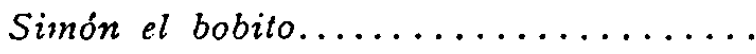

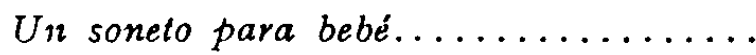

A Ladislao Errázuriz................

Lastarria.

Sonelos americanos $I \ldots \ldots \ldots \ldots \ldots \ldots$

Sonetos americanos $I I \ldots \ldots \ldots \ldots \ldots \ldots$

La Revolución Francesa $I \ldots \ldots \ldots \ldots$......

La Revolución francesa $I I \ldots \ldots \ldots \ldots \ldots$

La Revolución francesa $I I I \ldots \ldots \ldots \ldots$.

La Revolución francesa $I V \ldots \ldots \ldots \ldots$

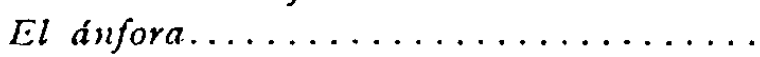

Rima rer
núcleo

Abrazada

Abrazada

Abrazada

Abrazada

Abrazada

Abrazada

Abrazada

Abrazada

Abrazada

Abrazada

Abrazada

Abrazada

Abrazada

Abrazada

Abrazada

Abrazada

Abrazada

Abrazada

Abrazada

Abrazada

Abrazada

Abrazada

Abrazada

Cruzada

Abrazada

Abrazada

$\triangle A A B$

CCCB

Cruzada

Cruzada

Cruzada

Cruzada

Cruzada

Cruzada

Cruzada cdc cdc

Rinta

tercetos

cdc dcc cdc dee cdc dee cdc dee cdc dee cdc dee cdc ded cde ded cdc cdc cdc eed cdc ded cdc ded cdc ded cdc ded cde ded cdc ded cdc dee cde cde cdc dee cdc dee cdc dee cde cde

cde ded cdc ded cdc dcd ccd eed

cde dee ccd eed cdc ded cdc dicd cdc ded ccd eed cdc derl 


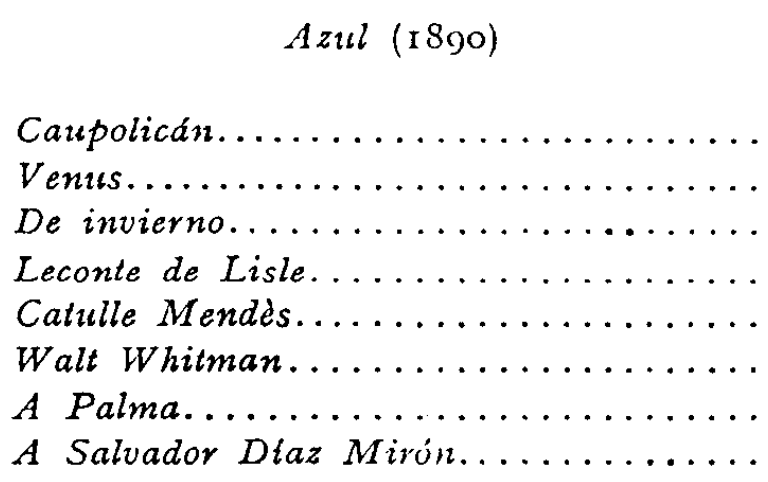

De I 890 a I 893

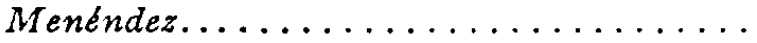
Maximo Soto Hall.

Colombia.

\section{Prosas profanas (1896)}

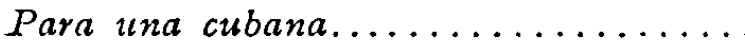

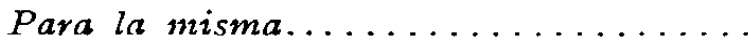

Margarita.

Mia...

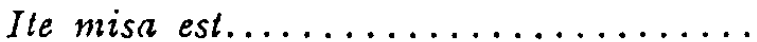

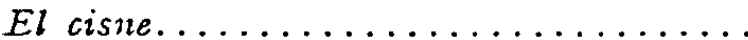

La dea.

La espiga.

La' fuente.

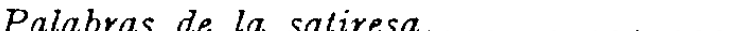

La anciana.

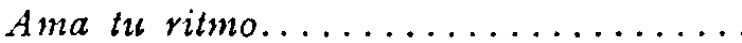

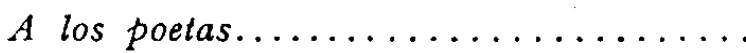

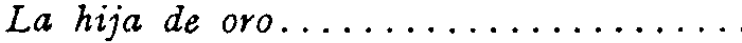

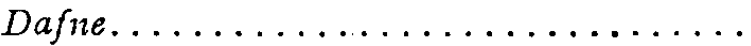

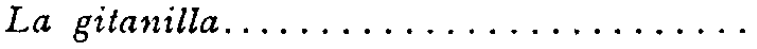

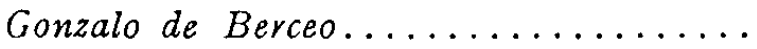

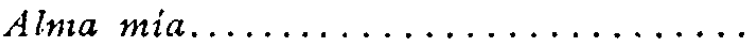

"Yo persigon.................

Cantos de Vida y Esperanza (1905)

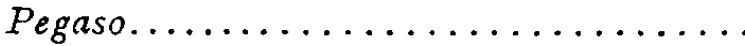

Los cisnes.

La dulzura del Angelus.
Rinla I er núcleo

Cruzada

Cruzada

Cruzada

Cruzada

Cruzada

Cruzada

Cruzada

Cruzada

Alterna

Abrazada

Abrazada

Abrazada

Abrazada

Cruzada

$A B B A$

$A B A B$

Cruzada

Cruzada

Cruzada

Cruzada

Cruzada

I $-4-6-8$

2-3-5-7

Alrazada

Abrazada

Abrazada

Cruzada

Alorazada

Abrazada

Abrazada

Abrazarla

Abrazada
Rima

tercetos

ccd ccd clc ded crlc ded ccd eed cod eed $\mathrm{cdc}$ ded cdc colc ccd eed

Aiteria clc ded chle rled

-.. cod eed

-... cal eed

... cale ecd

-.- ccl cerl

--- cod ced

-.. cerl ecd

... colc cdlc

-.- ccd eed

..- ced eed

-.. cde crlc

... ced eed

... cilc ded

... cde ded

-.. crle ded

-.. cde ded

-... coll eed

-.. colc ded

-.- ccrl eed

-.- ccd eed
Abrazada Abrazada

A BBA

$\triangle A B B$
-.. cole cile

-... colc colc

-.. crle cile

-.. che cile. 


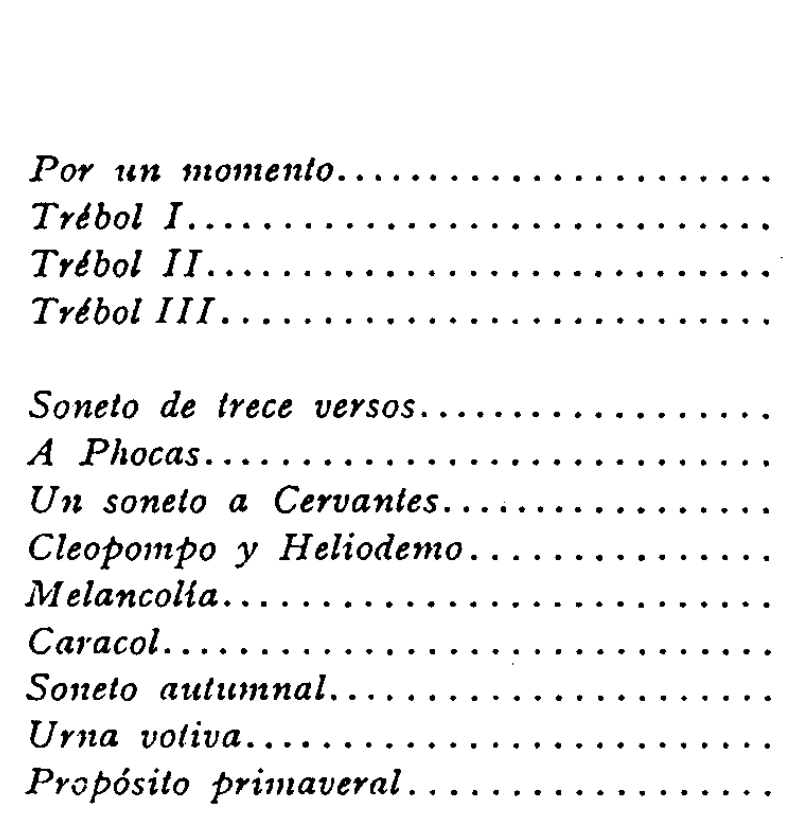

El canto errante (1907)

Israel. ...................

A Francia.

Libros extraños.....................

"Esquela a Charles"..................

"Helda".........................

"Soneto a Valle»....................

Tant miettx....................

Lirica........................

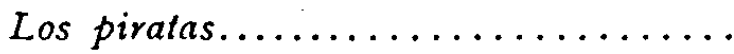

Poema de otoño (rgro)

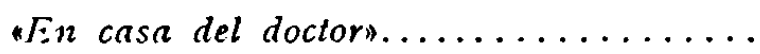

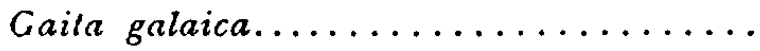

$$
\begin{aligned}
& \text { Rima I. er } \\
& \text { uúcleo }
\end{aligned}
$$

Abrazada

Abrazada

Abrazada

$A B A B$

$\mathrm{ABBA}$

Abrazada

Abrazada

Abrazada

Abrazada

Abrazada

Abrazada

Abrazada

Abrazada

Abrazada
Rima

tercetos

-.. cde catc

-... cde ded

--- cdc ded

-.- ccd eed

-.- cdc cl

-.- cdle ded

... cdc ded

-.- ccd eed

--- ccd eed

-.. ccd eed

--- cle cle

--- ccd eed

-.- ccd eed

Abrazada

--- cclc ded

Cruzada --- ccd eed

Abrazada

-.. colc ded

Cruzada

-.. ccd eed

Abrazada

--- ccd eed

Cruzada

-.. cdc ded

Abrazada

--- cdc ded

Abrazada

-.- ccd eed

Abrazada

-.- ccd eed

Abrazada

--- ccd dee

Abrazada

-.- ced ccd

\section{De 1893 a 1914}

1 $893-1897$

Wagneriana (I) Lohengrin..........

Albrazada

-..- ccd eerl

Wagneriana (II) Parsifal.............

Abrazada

--- ccrl eerl

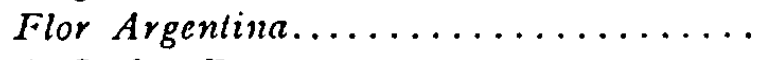

Cruzada

--- ccd eed

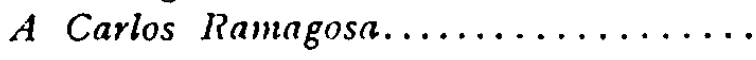

Cruzada

-.- ccd dee

"Toasin.

Cruzada

-.- ccd eed

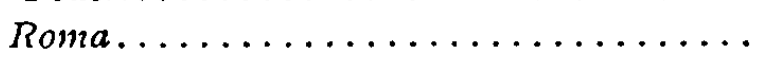

Abrazada

-.. cdc ded

A Suzelle.

Abrazada

... ced eed 


\begin{tabular}{|c|c|c|}
\hline & $\begin{array}{c}\text { Rinua }{ }^{e r} \\
\text { nuicleo }\end{array}$ & $\begin{array}{c}\text { Rima } \\
\text { tercetos }\end{array}$ \\
\hline \multicolumn{3}{|l|}{ I 898-1907 } \\
\hline España.. & Abrazada & --- cde ded \\
\hline A Bolivia...... & Abrazada & ... cod eed \\
\hline A Juan Ramón..... & Abrazarla & -.- cod eed \\
\hline Amado Nervo............ & Cruzada & --- cde rlcd \\
\hline La tortuga de oro $\ldots \ldots \ldots \ldots \ldots \ldots$ & Abrazada & --- ccrl dee \\
\hline Ante el David de Miguel Angel.... & Abrazada & --- cde ded \\
\hline "Toast" a D. J. Sierra............... & Abrazada & --- cde ded \\
\hline Flora............. & Abrazarla & --- ccil eed \\
\hline A Guillermo Andrei'e..... & Abrazada & -- cde ded \\
\hline
\end{tabular}

Del chorro de la fuente

I908-19ro

"Tras los cuornos de Júpitern...........

Cruzada

--- coll eed

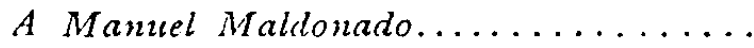

Abrazada

-.- cde dee

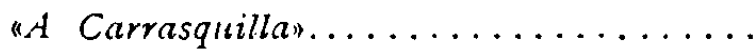

Cruzarla

Para Alice de Bolaños (I)...........

Abrazalla

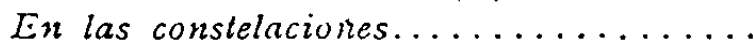

Cruzada

-.- ced ecd

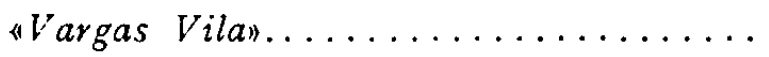

Abrazada

Toisón.

Cruzada

... col ede

"L.o que habla en el silencion..........

Cruzada

-.- coll eed

-.- cde ded

--- cole erle

-..- cde ded

\section{9 II I I I 4}

Triptico de Nicaragua (I)............

Abrazadia

--- crle dert

Triplico de Nicaragua (II)............

Abrazada

--- crle rlat

Triptico de Nicaragua (III)...........

Abrazarla

-.. crle derl

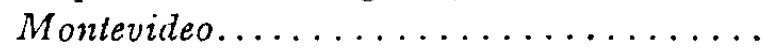

Abrazacla

-.. corl cerl

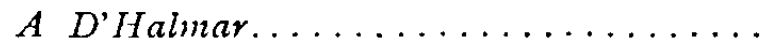

Cruzada

... cad cerl

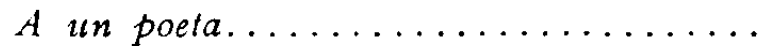

Abrazada

-.- ced ced

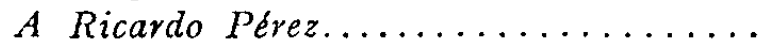

Abrazada

... crle dicl

"Puesto que tí me dices".............

ABBA

$B A B A$

-.. crle rlert

A Francisca (I) $\ldots \ldots \ldots \ldots \ldots \ldots$

Abrazada

-.- col eed

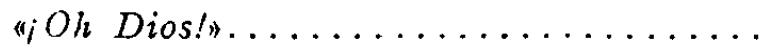

Abrazada

-.. colc erle

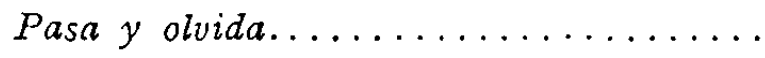

Cruzada

-.- ced eerl

Para ir al Azul...

Abrazada

-.. cede ded

\section{Navidad I9I4}

Soneto Pascrial.

Abrazarla

-.. cerl eed 
Resumen: respecto del primer núcleo, los ocho primeros versos del solleto:

a) Esquema tradicional $A B B A: A B B A$

Son 84 los sonetos con este esquema. Es muy significativo que los 22 primeros - hasta I886 - lo sigan; de I886 a I889 sólo dos de los (liez sonetos escritos por Darío presentan esta disposición rítmica ( $U n$ soneto para bebé y $A$ Ladislao Errazuriz).

Los ocho sonetos de $A z u l$ tienen rima cruzada. De los I9 sonetos de Prosas profanas, nueve siguen este esquema y dos son casos especiales, que estudiamos aparte. De los 9 sonetos de El canto crrante seis siguen este esquema; exactamente igual los dos sonetos de Poema de otoño; cn cuanto a los recogidos en la Obra dispersa, 27 sonetos ofrecen este esquema a partir de 1890 , fecha de publicación de la segunda edición de Azul.

b) Esquema de rima cruzada: $A B A B: A B A B$

Hasta I 886 ningún soneto hay con esta disposición, de 1886 a ISS9 de dicz sonetos, siete la siguen, como asimismo los ocho de $A z u l$, ocho también de Prosas profanas, tres de Canto errante y once de la Obra dispersa, a partir de I8go.

\section{c) Casos especiales}

El soneto Mia (p. 569) de Prosas profanas es el primero con una disposición especial en cuanto a la rima del primer núcleo: los cuatro primeros versos llevan la rima tradicional o abrazada, y los cuatro siguicntes, rima cruzada.

En cl mismo libro, Palabras de la satiresa (p. 6I6) presenta el siguiente esquema: $A B B A: B A B A$.

Iin Cantos de vida y esperanza, La dulzura del Angelus (p. 655) sigue cl esquemia de rima abrazada en los cuatro primeros versos, pero los cuatro siguientes riman $\triangle A B B$.

En el tercer soneto de Trébol (p. 66r) del mismo Jibro, hay rima cruzada en los cuatro primeros versos, y rima abrazada en los cuatro siguientes.

Y en la Obra dispersa, tres sonetos of recen una disposición especial: Lastarria (p. 885) con el esquema AAAB:CCCB; Menéndez, ya estudiado en uno de los apartados anteriores, y la composición Puesto que tí me dices (p. Io7?), el esquema ABBA:BABA. 
Los sonetos de Rubén presetan la siguiente disposición en cunnto a la rima de los ocho primeros versos: rima abrazada, 84 ejemplos; rima cruzada, 37 ejemplos y casos especiales, 7 ejemplos.

Respecto de los seis versos finales de los sonetos; y en primer lugar, de aquellos en que los tercetos tienen únicamente dos rimas:

a) Hasta $A z u l l$, tres esquemas:

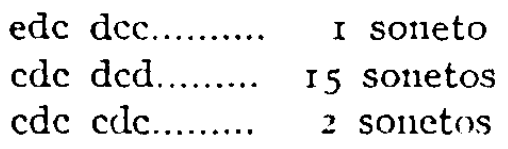

b) Desde $A z u l$, cuatro esquemas: ccd ccd........ 4 sonetos cdc $\operatorname{dcd} . . . \ldots . . .32$ sonetos cdc cdc........ 6 sonetos cdc ced........ I soneto

Con tres rimas, los sonetos siguientes:

a) Hasta $A z u l$, cuatro esquemas: cde dee........ ro sonetos cdc eed......... 2 sonetos cde cde........ 2 sonetos ccd eed......... 2 sonetos

b) Desde Azul, ocho esquemas: ccd eed........ 40 sonetos cde cde........ I soneto ccd dee........ 3 sonetos cdc dee......... 1 soneto ccd ede......... I soneto cdc ded......... I soneto clc edc........ I soneto cdc ede........ I soneto

d) Casos especiales

En La dulzura del Angelus (p. 655) el primer verso de los tercetos rima con el quinto y el sexto del primer núcleo.

\section{Conclusion}

Darío empleó indistintamente dos o tres rimas en los tercetos: Con dos rimas, 64 sonetos; con tres rimas, 59; casos especiales, 5 .

Los cuatro esquemas preferidos por nuestro poeta fueron: $A B B A$ : $A B B A$ cdc ded; $A B B A: A B B A$ ccd ced; $A B A B: A B A B$ cdc ded; $A B A B$ : $A B A B$ ccd eed. 
Melancolía, incluido en Cantos de Vida y Esperanza, es un soneto heterométrico; alejandrinos, $\mathrm{I}, 2,6,7,8,9$, 10, 13 y 14 ; endecasilabos, 3,4 y 5 , y heptasílabo, Ix. Un soncto de Cervantes, incluido en el mismo libro, se compone de versos endecasílabos y versos heptasílabos; como dice Oliver Belmás, es una silva de catorce versos, distribuidos estróficamente como un soneto. En casa del doctor Luis H. Debayle. - Toast, incluido en Poema de otoño y otros poemas, es un soneto en alejandrinos, con seis versos en estrambote, el único caso entre los sonetos de Darío.

$\mathrm{Y}$ por último, Roma no tiene a Rubén como único autor; los versos impares fueron compuestos por Lamberti y los pares por nuestro poeta en un café de Buenos Aires, en I $896^{1}$.

\section{Estructura temática y sintáctica ${ }^{2}$}

Mucho se ha debatido acerca de la unidad del soneto o de su composición policstrófica; problema muy estudiado, pero con subordinación, la mayoría de las veces del contexto a la estructura formal o viceversa. $\mathrm{Y}$ ambas cosas van unidas: el poeta expresa ideas o sentinicntos en un marco de unidad, el soneto, pero conformando el contexto a cláusulas poéticas, las estrofas. Porque la unidad primaria conceptual, y en muchos casos sintáctica, es la estrofa, aunque, efectivamente, se encuentren ejemplos de sonetos en que este principio no se cunple; pero estos ejemplos siempre tendrán alguna característica especial. Véase el siguiente, en que esa característica es una acentuada correlación:

1 Antonio Olivir Brimis, Ese olro Rubén Dario, p. 374. Lin este tomeo a lo culto dio Rubén el pie forzado y Antonio Lamberti estuvo a la altura de las circunstancias. Buen poeta I amberti para contender líricamente con Darío. Los clos merecieron el premio del vaso labrado en asta, con que se galardonaba a "los líricos clísicos. Los dos libaron toda la noche para celebrar el combate. Iros dos, árbitros. Porque salieron casi a minuto por verso. iJuego noble y preclaro el del soneto "Roman!...

2 Aunque hagamos uso de algunos puntos básicos de la técnica estructuralista, no pretendemos hacer un análisis estructural de los sonetos de Rubén, ya que hacerlo por completo equivaldria a multiplicar estas páginas por el número rle sonetos que escribió nuestro poeta. Véase como modelo de análisis estructural el artículo de N. Ruwrix, Analyse structurale d'un poème français. Linguistics, ro64, núm. $3, \mathrm{pp}, 62-8_{3}$. 
"Tant mieux"

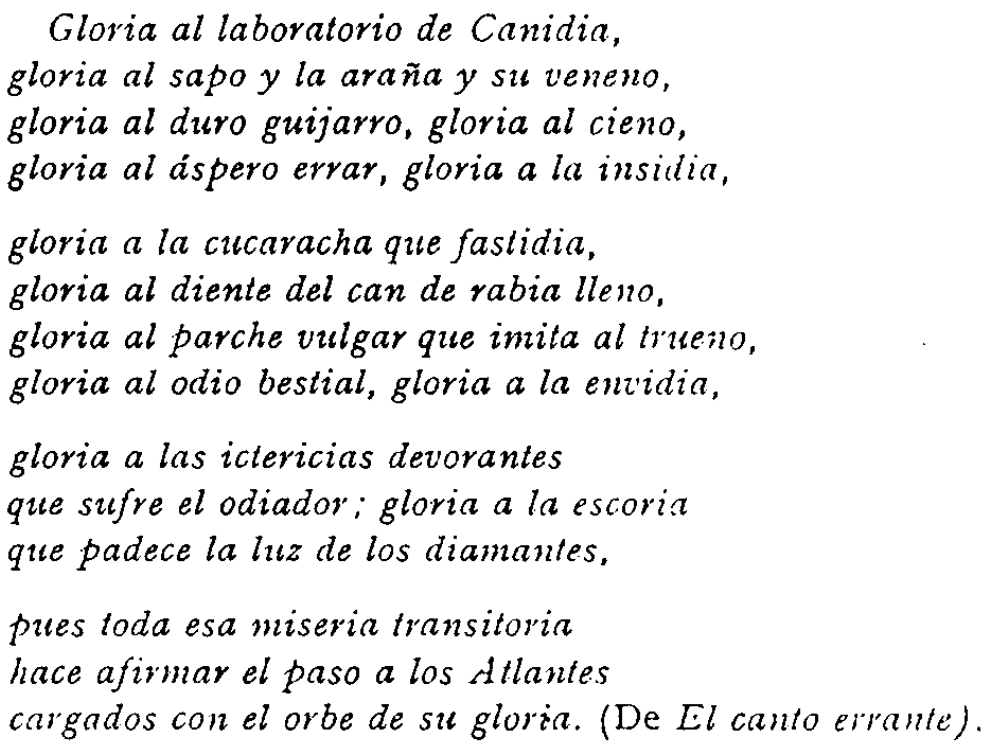

Rubé11 en los sonetos, como los poetas de nuestro Siglo de Oro y los buenos sonetistas posteriores, tiene muy en cuenta la estrofa; $y$ en la gran mayoría de los sonetos, después de cada estrofa hay pausa larga, aquella que es necesaria y requieren los elementos de ejecución de la lengua hablada, representada normalmente por punto o punto y coma, y que divide sintácticamente oraciones diferentes, $y$, temáticamente, $o$ son conceptos diferentes los que expresan cada una de sus partes o, semejante la base conceptual, hay diferencias cualitativas: de enriquecimiento, de acentuación expresiva, etc.

A partir del plano temático, fonético y morfosintáctico de los sonctos de Därío, puede llegarse a las siguientes conclusiones:

I. ${ }^{\circ}$ En cuanto al contexto, los sonetos de Rubén estarían dividiclos en dos o tres partes: en unos casos, de I a 8 y de 9 a I 4 ; en otros, de 1 a 4 , de 5 a 8 y de 9 a I 4 . En los sonetos divididos en dos partes, las diferencias entre ambas es patente: diálogo en los tercetos cuando los cuartetos han sido narrativos, o viceversa, aunque en muchas menos ocasiones; acentuación expresiva en los últimos versos sobre los primeros; aparición de un epifonema, interrogación, etc., de un número variable de versos, que cierra la composición; personalización en la ultima parte de las imágenes objetivas de la primcra... En los sonetos divididos en tres partes, las diferencias entre el primer cuarteto y el segundo son más difusas; no olvidemos que el tema es el mismo a través de la composición y lo que liace Rubén es dibujar tres cuadros encadenados, variaciones sobre el tema único. Esta división no se cumple en algunas ocasiơnes, y, en lugar de acabar el primer núclco en el verso cuarto, a reccs lo hace en el tercero o 
en el quinto, y en tres casos tampoco se da en el verso octavo sino en el noveno. Pero esto va unido a la estructura sintáctica cle los sonctos.

2. ${ }^{\circ}$ Fonética y sintácticamente, los sonetos de Rubén siguen el tradicional esquema en que cada verso es una uniclad con pausa versal al final del mismo, y estrofas con una estructura sintáctica cerrada y pausa estrófica al final de ellas. Pero hay, en primer lugar, muchos sonetos con pausas internas (además de las obligadas por los versos compuestos), que suponen una alteración de la estructura general de la composición, faltando las pausas versales y produciéndose encabalgamiento entre versos, y algo más importante, ya que una de las condiciones que debe reunir una estrofa, además del axis rítmico, de un número determinado de rimas y de un sistema estructurado de versos, es una estructura sintáctica determinada, es decir, que "el enunciado completo coincida con la pausa estrófica” 1; sin embargo, en Rubén hay sonetos cu que esto no se cumple: la pausa, después del primer cuarteto, no es larga, como fonéticamente debe ser la estrófica, dándose incluso encabalgamicnto estrófico, como en La dulzura del Angelus (p. 655), Cleopompo y' Heliodemo (p. 672) y A'DHalmar (p. Io7o). Veamos este último:

Como Pindaro tiende, hacia el viento que sopla,
la vela de sut nave, que es una carabela
de Cortés, por audaz, y de Constantinopla,
de París y de la India. Su palabra que vuela
sutilmente, recuerda la más cálida copla
de España. Su ascendencia un gran misterio vela.

Sintácticamente, este soneto cerraría su primer núcleo con India, en lugar de hacerlo con vucla; la oración que vuela sutilmente no permite la pausa entre el verbo y el adverbio, ya que es un sirrema ${ }^{2}$, por lo que se produce un encabalgamiento sirremático ${ }^{3}$.

İ̀n relación con el último núcleo del soneto, formado por los tercetos, hay algo importante y doncle es necesario detenerse, ya que encontramos, en muchísimos casos, un número variable de versos que son un colofón del poema, diferente del contexto anterior y representado por medio de un epifonema, una interrogación o simplemente por una oración simple o compuesta y que a modo de broche, resume, con una gran intensiclad lírica, toda la composición. Algunos ejemplos:

1 Antono Quir,Is, Métrica Española, Madrid, Alcalá, i969, pp. 87-88.

2 RAFAEI DE BALnfN, Sistema de ritmica..., p. 132 con nota 92.

3 ANTONIo Quiris, La estructura del encabalgamiento en la métrica española, Marlirl, C. S. I. C., Anejo I.XXVII de RFE, r964, pp. 95-ro.3 especialnnente. 
Iipifonema: con 6 versos:

¿Yo soy el caballero de la humana energia, yo soy el que presenta su cabeza triunfante coronada en el laurel de Rey del día;

domador del corcel de cascos de diamante voy en un gran volar, con la aurora por guía. adelante en el vasto azur, siempre adelante! (Pegaso, p. 639).

Con cuatro versos:

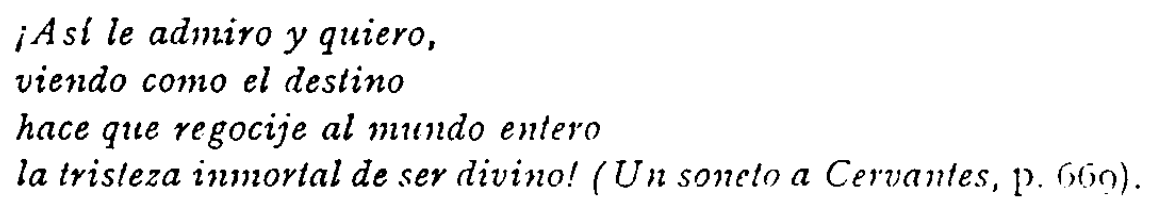

Con tres versos:

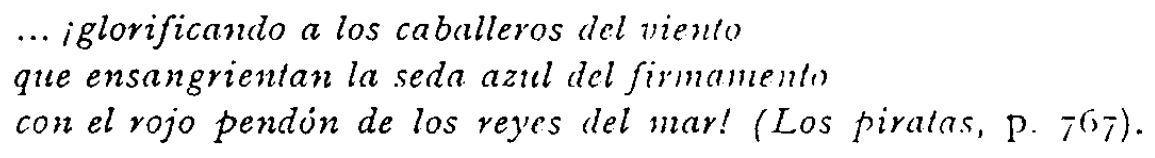

Con dos versos:

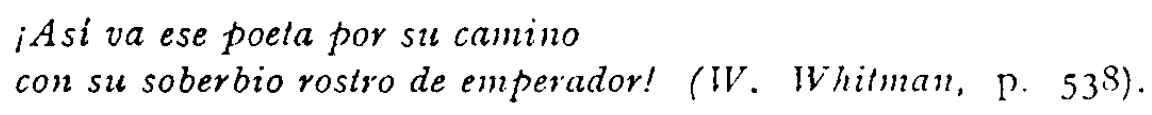

Con un verso:

... ilevantemos los ojos y juntemos las manos! (Para ir al .1zul, p. 100.1).

Con menos aún de un pie versal aparece el epifonema cn varias ocasiones:

... y una fuente soberbia, ila Rotonda! (Ante el David de Miguel Ang!l, p. Ioо.).

Interrogación: Con dos versos:

¿No has de ser entonces mía hasta la muerte? (Mla, p. $\left.5^{(6)}\right)$.

Con un verso:

¿No oyes caer las gotas de mi melancolia? (Melancolia, p. 675).

\section{Paréntesis:}

(El caracol la forma tiene de un corazin). (Caracol, p. 679). 
Iil soneto es, casi sicmpre, cn Rubén Darío una composición en tres tempos: en el primero, se destaca el objeto, el cencepto o el sentimiento de una forma pausada, cadenciosa; en el segundo, aumenta la cmotividad, subjetivándose generalmente lo que antes había sido objetivo, para, en muchos casos, scr el último o los últimos versos una cumbre emotiva, sentida con más intensidad. El soneto sería un templo griego de catorce columnas, como el mismo Darío dice, pero dividido en dos plantas: la primera, compuesta por las ocho primeras colunnas, y la segunda, en cuatro o cinco, para convertirse las dos últimas, o la última, en un frontón clásico, que quisiera elevar su vértice al cielo... O como una sinfonía en tres tiempos: cadenciosa, plácida, suave la primera; plena de vida y de fuerza, la segunda; y un "allegro molto final» o una cadencia melancólica y llena de belleza, la tercera.

I,UCIANO García T,ORINAZO 